Noname manuscript No.

(will be inserted by the editor)

\title{
Dual-band Dielectric Light-harvesting Nanoantennae Made by Nature
}

Julian Juhi-Lian TING

to the memory of my father.

the date of receipt and acceptance should be inserted later

\begin{abstract}
Mechanisms to use nanoparticles to separate sunlight into photovoltaic useful range and thermally useful range to increase the efficiency of solar cells and to dissipate heat radiatively are discussed based upon lessons we learnt from photosynthesis. We show that the dual-band maxima in the absorption spectrum of bacterial light harvestors not only are due to the bacteriochlorophylls involved but also come from the geometry of the light harvestor. Being able to manipulate these two bands arbitrarily enables us to fabricate the nanoparticles required. Such mechanisms are also useful for the design of remote power charging and light sensors.
\end{abstract}

Keywords dielectric resonator antenna · light-harvesting complex · energy transfer $\cdot$ photosynthesis $\cdot$ metamaterial

PACS wave optics 42.25.-p · biomolecules 87.15.-v

De-Font Research Institute, Taichung 40344, Taiwan, R.O.C.

E-mail: juhilian@gmail.com URL: http://amazon.com/author/julianting 


\section{Introduction}

Waste heat disposal is an important issue in everyday life, ranging from our personal computers to power plants. Recently, two seemingly unrelated studies in this topic actually employed the same mechanism which our previous study on bacteria light harvestors can provide theoretical guidelines. The first one concerns about the conversion efficiency of solar cell, while the second one is a general waste heat dissipation method [1, 2].

Solar-cell production is a mature industry today. Trying to improve the efficiency of solar cells with the material used is as difficult as trying to raise the critical temperature of high-temperature superconductors, not even to mention there is a Shockley-Queisser limit 3. However, the conversion efficiency of solar cells will be reduced by $0.4-0.5 \%$ for each one degree Celsius raised above their normal working temperature, i.e., $25^{\circ} \mathrm{C}$, 4, 5, 6, which is unavoidable because some frequencies of light are not useful for the solar cell and will be converted into heat eventually. Various methods trying to reduce the working temperature have been proposed 77. An ingenious method to pre-filter the sunlight involves spreading of nanoparticles into water to separate the light into a photovoltaically useful range and a thermally useful range has been proposed [1. A similar general cooling method uses the reversed mechanism, which embeds particles into polymethylpentene films to enhance the radiation, was also proposed 2. Although these authors arrived at their proposal mainly through experimental trial and error, there exist theories to design what kind of particles should be used to achieve the best performance [8, 9].

One can readily recognise that the particles used by the latter authors are antennae, but what the former authors called filters are also better described as antennae, in particular, nanoantennae, which are devices designed to convert free radiation efficiently into localised energy, such as with lenses and mirrors, or vice versa 10. The theory of antennae sums up individual effects of the lightmatter interaction, in which the most important parameter is the geometry that produces the retarded effects. Receiving nanoantennae may confine incident light into subwavelength region. Optical antennae pose special problems because of their small size and the plasmon resonances of metal. A consideration of nanoantennae as solar energy collectors has appeared previously [11.

Many effective light absorbing molecules are made in nature. Chlorophylls interact with light stronger than others because they consist of alternating chemical double bonds and densely packed to enhance the absorption cross section. The structures of many bacteria light-harvesting complexes (LH) made of bacteriochlorophylls have been solved after McDermott et al. solved the structure of Rhodopseudomonas acidophlla in 1995 [12. Two of them are shown in the Table 1.

Understanding the light harvestor made by nature can not only help us to fabricate voltaic solar cells but can also help us to manufacture optical wireless power chargers [13] and make better electromagnetic wave sensors [14. Wireless power transfers, at this moment, work not only under microwave frequencies, if not radio frequencies, but also only under designed non-physiological condition 15 , 16. 17, 18. However, medical applications require the power chargers to work under flexible physiological condition [16]. The next generation wireless power transfer we discuss, will use incoherent light as the nature use, which is sustainable 


\begin{tabular}{lccc}
\hline protein & PDB ID & symmetry & cartoon \\
\hline LH2 B800-850 from Rhodopseudomonas acidophila $[24]$ & $1 \mathrm{NKZ}$ & $\mathrm{C} 9$ & \\
LH2 B800-850 from Rhodospirillum molischianum [25] & 1LGH & $\mathrm{C} 8$ & \\
\hline
\end{tabular}

Table 1 Various light harvestors II with structural symmetry. More can be found in PDB.

and renewable, and is robust and, in particular, is working under physiological condition.

In two previous papers, the non-reciprocal properties and the dipole properties of the bacteria light harvestors, LH1-RC/LH2 complexes, were considered by an infinitesimally thin loop antenna as a first approximation [19, 8]. Even though that loop antenna considered is too idealized, it can explain many phenomena and natural design physically without ad hoc parameter. However, even though the resonance frequency can be adjusted by various methods [20, a loop-antenna has only one resonance frequency, whereas a real light-harvesting complex shows not only a band of absorption frequency but a dual-band of absorption spectrum, with one in a region about $850-860 \mathrm{~nm}$ and the other near $800 \mathrm{~nm}$, which are generally attributed to the resonance frequencies of the bacteriochlorophylls involved 21, 22. There are indeed many methods to make a dual-band antenna [23], but the simplest way can be employed by the nature seems to come from the geometry of the light harvestor. We will show that there are geometrical reasons for these two maxima by extending our analysis to consider the finite thickness of the antenna. Being able to manipulate these two bands arbitrarily enable us to fabricate the nanoparticles required.

To simplify the issue, we will consider a LH2 system, without the feeding line and the reaction centre of the LH1 system, in the following. A LH2 consists of nine units for Rhodopseudomonas acidophila of inner diameter $36 \AA$ and outer diameter $68 \AA[12,24]$. The width is roughly double the height of the molecule. The dimensions of the molecule are obtainable using the data from the Protein Data Bank (PDB, http://www.rcsb.org/pdb/). Such systems are exactly what Hjerrild et al. and Zhai et al. considered, except that nature made the light harvestors with a few atoms whereas the following discussion considers the particles with bulk material as the previous authors. Because the light harvestors have random orientation, it would be better to consider their interaction with unpolarized light. The energy received by such antennae will be dissipated as heat into surrounding water. 


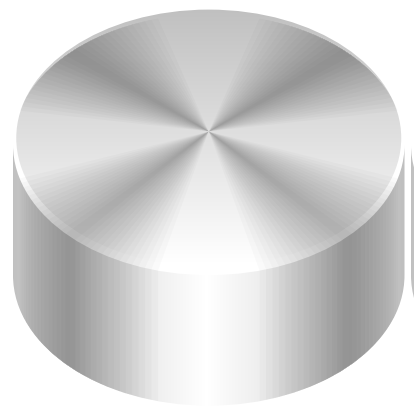

(a)

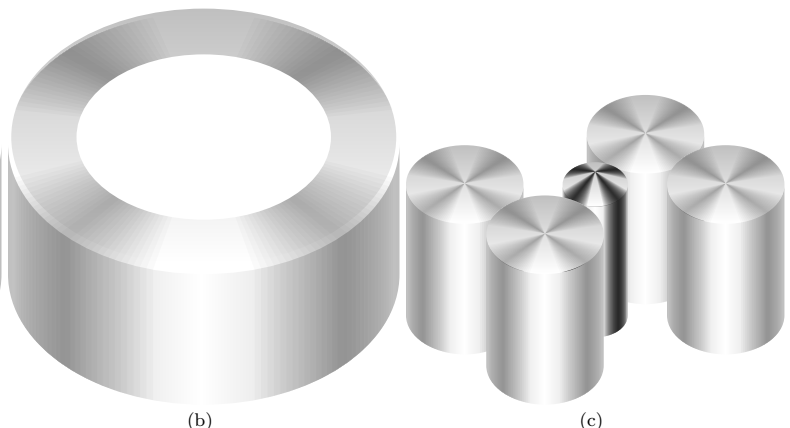

(b)
Fig. 1 Successive approximation of the bacterial light-harvesting antenna: (a) cylindrical approximation, (b) dielectric shell (ring) approximating an antenna, (c) four-element dielectric antenna with centre feed line.

\section{2 dielectric light harvestor}

Optical nanoantennae have been constructed in 2005 [26, 27]. Albeit most nanoantennae are as yet made of conductors [28, 29, at the frequency of solar light, metals are no longer perfect conductors hence the electric fields can penetrate inside, and dielectrics work better. Paraphrasingly, the conductivity turns out to be less relevant when the wavelength is reduced. Dielectric antennae are at present widely used in microwave engineering, optical-fibre engineering and, in particular, in mobile telephones [30, 31, 29. A dielectric antenna functions like an acoustic resonator; the electrons bounce back and forth inside the cavity, which is consistent with the exciton theories. All-dielectric nanoantennae have been fabricated recently 32 .

Presently people use conductor coated with dielectric to make such antenna, which is unnecessary. Using a dielectric material to build the light harvestor has many advantages [33, 34]:

- the dimension of the antenna can be diminished by a factor $1 / \sqrt{\epsilon_{r}}$ relative to the corresponding metallic one because the dielectric wave length is smaller than the free-space wavelength,

- relatively large bandwidth,

- low or no loss at optical frequencies,

- free from damage of high power or local heating [35],

- have less detuning when placed close to another object,

- compatibility with complementary metal-oxide semiconductor fabrication processes.

Symmetry breaking of the electric field in space facilitates the radiation of the antenna [36, which provides a third reason for the mysterious opening at the LH1-RC complex and explains the elliptical shape of the complex.

We made successive approximations for the light harvestor in Figure 1. The first antenna, Figure 1 (a), is a cylinder, which can be solved analytically, but the boundary condition that we consider differs slightly from standard engineering practice because in most antenna applications the dielectric material is placed on top of a magnetic conducting plate, whereas in photosynthesis the dielectric 
material is placed in a solvent. This does not cause much difficulty to apply the solutions found in the literature. Using the method of image, we simply consider a cylinder of double-height, $2 h$, so that the solution for an antenna placed on top of a magnetic conducting plate can be applied. The second one, a dielectric ring (or shell) antenna, as shown in Figure 1(b), can also be solved analytically with a superposition principle. The third one shown in Figure 1 (c) generally requires numerical methods of solution 37 .

We consider first a solid cylindrical dielectric material of diameter $a$ extending in direction $z$ from $-h$ to $h$ in a vacuum. Fields at the surface of a region of large relative permittivity satisfy approximately an open-circuit boundary condition, i.e. the normal component of the electric field and the tangential component of the magnetic field are zero [38. The resonance frequencies of least order read [33]:

$$
\begin{aligned}
f_{0}\left(H E M_{01}\right) & \approx \frac{c 6.323}{2 \pi a \sqrt{\epsilon_{r}+2}}\left[0.27+0.36\left(\frac{a}{2 h}\right)+0.02\left(\frac{a}{2 h}\right)^{2}\right] \\
f_{0}\left(T E_{01 \delta}\right) & \approx \frac{c 2.327}{2 \pi a \sqrt{\epsilon_{r}+1}}\left[1+0.2123\left(\frac{a}{h}\right)-0.00898\left(\frac{a}{h}\right)^{2}\right] \\
f_{0}\left(T M_{01 \delta}\right) & \approx \frac{c}{2 \pi a \sqrt{\epsilon_{r}+2}}\left[3.83^{2}+\left(\frac{\pi a}{2 h}\right)^{2}\right]^{1 / 2} \\
f_{0}\left(T E_{011+\delta}\right) & \approx \frac{c 2.280}{2 \pi a \sqrt{\epsilon_{r}+1}}\left[1+0.7013\left(\frac{a}{h}\right)-0.002713\left(\frac{a}{h}\right)^{2}\right]^{1 / 2}
\end{aligned}
$$

with $\epsilon_{r}=\epsilon / \epsilon_{0}$ been the relative permittivity, and $c$ is the speed of light. As we stated at the beginning, our $a / h \approx 2$ is well within the valid range of the approximation, which controls the bandwidth of the antenna 39 . Depends upon the relative permittivity, at a certain ratio of $a / h$ the bandwidth increased due to overlapping of two resonant modes. We use $h=34 \AA, a=68 \AA$ and $\epsilon_{r}=1000$ to obtain the values shown in the first row of Table 2, but this result is not yet what we want. What we seek is a shell (or ring) shape, which can be effected through superposition of two cylinders of different radius [40. The modes of a smaller cylindrical dielectric material of diameter $b$ with the same height $2 h$ can be obtained similarly and are shown in the second row of Table 2, The superposition produces an antenna with two maxima in the absorption spectrum, which is called a dual-band antenna, as the bacteria light harvestors also have two maxima in their absorption spectrum [41. These numbers are roughly what we require, as the relative permittivity for the light harvestor do not exist 42 .

The relative permittivity appears to be spurious high, because we are using radio frequency formulae for bulk material properties. At optical frequencies even matal is no longer perfect conductors. The skin depth $d=\lambda /\left(4 \pi \sqrt{\epsilon_{r}}\right) \approx 20 \AA$. Electricmagnetic fields have fully penetrated the antenna of thickness $30 \AA$. The volume currents and reduced wavelength for optical antennae need to be adjusted by about $20 \%$ [3]. Furthermore, the nature is not using bulk material. However, even without these adjustments we have already shown it is possible to produce the dual-band spectrum. A similar circular polarized antenna excited with a tilted modified square slot has been studied 23 .

For two reasons it would be better to divide the light harvestor into sub-units as shown in Figure 1 (c):

- For a dielectric antenna to achieve a satisfactory efficiency, proper modes must be excited by sunlight, as shown in Figure 5 of $\mathrm{Yu}$ et al. [44. Modules of bacteriochlorophyll appear at nodes of the electric field [44, 45]. / 


\begin{tabular}{lcccc}
\hline radius/mode & $T E_{01 \delta}$ & $H E M_{11 \delta}$ & $T M_{01 \delta}$ & $T E_{011+\delta}$ \\
\hline $68 \AA$ & 0.717 & 0.912 & 1.099 & 1.172 \\
$36 \AA$ & 1.185 & 1.235 & 1.75 & 1.61 \\
\hline
\end{tabular}

Table 2 Various mode resonance frequencies/ GHz with $h=34 \AA$ and $\epsilon_{r}=1000$.

- A ring that is constructed from sub-units corresponds to an interconnection of inductors interleaved by capacitors works better than a continuous one, because it guides the flow of displacement current better [46]. Furthermore, it has a band-stop frequency response that rejects a certain range of frequency.

A traditional theory of photosynthesis describes this part according to exciton theory without detail. In view of this second reason, the light harvestor built by previous authors apparently has room for improving their efficiency [44, 1, 47].

\section{Summary}

Historically, many authors tried to calculate spectroscopic properties from some theoretical models as the spectrum is readily accessible experimentally 42 . In this work, we show that it is possible to place two maxima of the spectrum at the position desired by the classical electrodynamics model we proposed. An important difference between radiowave and optical antenna is at the way the receiver is connected to the antenna as shown by the LH1 designed by nature. We can also have a multi-element multi-layer model, which resembles the light harvestor even better, with radiation or receiving pattern achieving a monopole-like efficiency [48. Soren et al. considered a Sierpinski carpet patterned cylindrical Dielectric Resonator Antenna made by Teflon, which resemble our light-harvesting antennae even better 49 .

The nanoparticles that Hjerrild et al. considered are disk-shaped metallic particles of diameter roughly $100 \mathrm{~nm}$, which exploit plasmon resonance. We show that the optimal shape of the particles should be toroidal instead of discotic or nanorods for the infrared range [8, 19]. These authors further pointed out that silver is the best material to use, but that is quite expensive. They considered the material used to be important for the frequencies of absorption, and mentioned that the ratio of surface to volume of these nanoparticles makes the particles susceptible to damage from high power. Therefore they coated their metallic disk with dielectric. We have shown that full dielectric antennae can be used to avoid such high-power damage, which is actually the best material at such a scale, and that the frequency of absorption can be tailored with the radii of the toroidal particles.

\section{References}

1. N.E. Hjerrild, S. Mesgari, F. Crisostomo, J.A. Scott, R. Amal, R.A. Taylor, Sol. Energy Mater. Sol. Cells 147, 281 (2016). DOI 10.1016/j.solmat.2015.12.010

2. Y. Zhai, Y. Ma, S.N. David, D. Zhao, R. Lou, G. Tan, R. Yang, X. Yin, Science (80-. ). 355(6329), 1062 (2017). DOI 10.1126/science.aai7899

3. W. Shockley, H.J. Queisser, J. Appl. Phys. 32(3), 510 (1961). DOI 10.1063/ 1.1736034 
4. J.J. Wysocki, P. Rappaport, J. Appl. Phys. 31(3), 571 (1960). DOI 10.1063/ 1.1735630

5. D. King, J. Kratochvil, W. Boyson, in Conf. Rec. Twenty Sixth IEEE Photovolt. Spec. Conf. - 1997 (IEEE, 1997), pp. 1183-1186. DOI 10.1109/PVSC.1997. 654300

6. O. Dupré, R. Vaillon, M. Green, Sol. Energy Mater. Sol. Cells 140, 92 (2015). DOI 10.1016/j.solmat.2015.03.025

7. J. Oh, B. Rammohan, A. Pavgi, S. Tatapudi, G. Tamizhmani, G. Kelly, M. Bolen, IEEE J. Photovoltaics 8(5), 1160 (2018). DOI 10.1109/JPHOTOV. 2018.2841511

8. J.J.L. Ting, J. Photochem. Photobiol. B Biol. 179, 134 (2018). DOI 10.1016/ j.jphotobiol.2018.01.011

9. P.K. Jain, Phys. Today 71(8), 10 (2018). DOI 10.1063/PT.3.3984

10. L. Novotny, B. Hecht, Principles of Nano-Optics (Cambridge University Press, Cambridge, 2006). DOI 10.1017/CBO9780511813535

11. D.K. Kotter, S.D. Novack, W.D. Slafer, P.J. Pinhero, J. Sol. Energy Eng. 132(1), 011014 (2010). DOI 10.1115/1.4000577

12. G. McDermott, S.M. Prince, A.A. Freer, A.M. Hawthornthwaite-Lawless, M.Z. Papiz, R.J. Cogdell, N.W. Isaacs, Nature 374(6522), 517 (1995). DOI 10.1038/ $374517 \mathrm{a} 0$

13. D. Elsheakh, in Microw. Syst. Appl., ed. by S.K. Goudos (IntechOpen, Rijeka, 2017), chap. 08, pp. $155-205$. DOI $10.5772 / 64918$

14. M. Watanabe, A. Nakamura, A. Kunii, K. Kusano, M. Futagawa, J. Phys. Conf. Ser. 660(1), 0 (2015). DOI 10.1088/1742-6596/660/1/012110

15. N. Shinohara, Wireless Power Transfer via Radiowaves. ISTE (John Wiley \& Sons, Inc., Hoboken, NJ, USA, 2013). DOI 10.1002/9781118863008

16. J.L. Miller, Phys. Today 67(8), 12 (2014). DOI 10.1063/PT.3.2464

17. N. Keskin, H. Liu, in 2015 IEEE 65th Electron. Components Technol. Conf. (IEEE, 2015), Dc, pp. 1828-1833. DOI 10.1109/ECTC.2015.7159848

18. J.S. Tsai, J.S. Hu, S.L. Chen, X. Huang, Adv. Mech. Eng. 8(2), 1 (2016). DOI $10.1177 / 1687814016632693$

19. J.J.L. Ting, p. 1702.06671 (2019)

20. A. Alù, N. Engheta, Phys. Rev. Lett. 101(4), 043901 (2008). DOI 10.1103/ PhysRevLett.101.043901

21. H.M. Wu, N.R.S. Reddy, G.J. Small, J. Phys. Chem. B 101(4), 651 (1997). DOI 10.1021/jp962766k

22. S. Georgakopoulou, R.N. Frese, E. Johnson, C. Koolhaas, R.J. Cogdell, R. van Grondelle, G. van der Zwan, Biophys. J. 82(4), 2184 (2002). DOI 10.1016/ S0006-3495(02)75565-3

23. D. Pathak, S.K. Sharma, V.S. Kushwah, Prog. Electromagn. Res. M 62(November), 123 (2017). DOI 10.2528/PIERM17092701

24. M.Z. Papiz, S.M. Prince, T. Howard, R.J. Cogdell, N.W. Isaacs, J. Mol. Biol. 326(5), 1523 (2003). DOI 10.1016/S0022-2836(03)00024-X

25. J. Koepke, X. Hu, C. Muenke, K. Schulten, H. Michel, Structure 4(5), 581 (1996). DOI 10.1016/S0969-2126(96)00063-9

26. P.J. Schuck, D.P. Fromm, A. Sundaramurthy, G.S. Kino, W.E. Moerner, Phys. Rev. Lett. 94(1), 017402 (2005). DOI 10.1103/PhysRevLett.94.017402

27. J.N. Farahani, D.W. Pohl, H.J. Eisler, B. Hecht, Phys. Rev. Lett. 95(1), 17402 (2005). DOI 10.1103/PhysRevLett.95.017402 
28. P. Bharadwaj, B. Deutsch, L. Novotny, Adv. Opt. Photonics 1(3), 438 (2009). DOI 10.1364/AOP.1.000438

29. F. Monticone, C. Argyropoulos, A. Alu, IEEE Antennas Propag. Mag. PP(99), 1 (2017). DOI 10.1109/MAP.2017.2752721

30. S. Yoon, C. Park, M. Kim, K. Kim, Y. Yang, in 2010 Asia-Pacific Microw. Conf. (2010), pp. 219-222

31. J. Lee, J. Lee, K. Min, Y. Cheon, IEEE Antennas Wirel. Propag. Lett. 13, 935 (2014). DOI 10.1109/LAWP.2014.2323066

32. Z. Li, X. Liu, N. Xu, J. Du, Phys. Rev. Lett. 114(14), 1 (2015). DOI 10.1103/ PhysRevLett.114.140504

33. R.K. Mongia, P. Bhartia, Int. J. Microw. Millimeter-Wave Comput. Eng. 4(3), 230 (1994). DOI 10.1002/mmce.4570040304

34. A.I. Kuznetsov, A.E. Miroshnichenko, M.L. Brongersma, Y.S. Kivshar, B. Luk'yanchuk, Science (80-. ). 354(6314), aag2472 (2016). DOI 10.1126/ science.aag2 242

35. R.C.J. Hsu, A. Ayazi, B. Houshmand, B. Jalali, Nat. Photonics 1(9), 535 (2007). DOI 10.1038/nphoton.2007.145

36. D. Sinha, G.A.J. Amaratunga, Phys. Rev. Lett. 114(14), 147701 (2015). DOI 10.1103/PhysRevLett.114.147701

37. D. Guha, Y. Antar, IEEE Trans. Antennas Propag. 54(9), 2657 (2006). DOI 10.1109/TAP.2006.880766

38. S. Ramo, J.R. Whinnery, T. Van Duzer, Fields and waves in communication electronics, 3rd edn. (Wiley, New York, 1994)

39. C.S. DeYoung, S.A. Long, IEEE Antennas Wirel. Propag. Lett. 5(1), 426 (2006). DOI 10.1109/LAWP.2006.883952

40. Y.X. Guo, Y.F. Ruan, X.Q. Shi, IEEE Trans. Antennas Propag. 53(10), 3394 (2005). DOI 10.1109/TAP.2005.856381

41. C.J. Law, A.W. Roszak, J. Southall, A.T. Gardiner, N.W. Isaacs, R.J. Cogdell, Mol. Membr. Biol. 21(3), 183 (2004). DOI 10.1080/09687680410001697224

42. R.G. Alden, E. Johnson, V. Nagarajan, W.W. Parson, C.J. Law, R.G. Cogdell, J. Phys. Chem. B 101(23), 4667 (1997). DOI 10.1021/jp970005r

43. L. Novotny, Phys. Rev. Lett. 98(26), 266802 (2007). DOI 10.1103/ PhysRevLett.98.266802

44. Y. Yu, V.E. Ferry, A.P. Alivisatos, L. Cao, Nano Lett. 12(7), 3674 (2012). DOI $10.1021 / \mathrm{nl} 301435 \mathrm{r}$

45. D. Soren, R. Ghatak, R.K. Mishra, D.R. Poddar, Prog. Electromagn. Res. B 60(July), 195 (2014). DOI 10.2528/PIERB14031306

46. A. Alù, N. Engheta, Phys. Rev. B 78(8), 085112 (2008). DOI 10.1103/ PhysRevB.78.085112

47. N.E. Hjerrild, R.A. Taylor, Phys. Today 70(12), 40 (2017). DOI 10.1063/PT. 3.3790

48. R.K. Chaudhary, K.V. Srivastava, A. Biswas, in 2011 Natl. Conf. Commun. (IEEE, 2011), pp. 1-5. DOI 10.1109/NCC.2011.5734715

49. D. Soren, R. Ghatak, R.K. Mishra, D.R. Poddar, J. Electromagn. Anal. Appl. 04(01), 9 (2012). DOI 10.4236/jemaa.2012.41002 